\title{
Lesions of the Periaqueductal Gray and Rostral Ventromedial Medulla Disrupt Antinociceptive but Not Cardiovascular Aversive Conditional Responses
}

\author{
Fred J. Helmstetter and Sheralee A. Tershner \\ Department of Psychology, University of Wisconsin-Milwaukee, Milwaukee, Wisconsin 53201
}

The presentation of an auditory stimulus that signals a noxious event such as foot shock results in the simultaneous expression of multiple aversive conditional responses (CRs), which include a transient elevation of arterial blood pressure (ABP) and an opioid-mediated form of hypoalgesia. Recent evidence suggests that the neural circuits responsible for the expression of these two aversive responses may overlap. In the present study, rats were trained using a Pavlovian fear conditioning paradigm in which white noise was repeatedly paired with shock. After training, groups of animals received electrolytic lesions centered in the dorsal or ventral periaqueductal gray (PAG) or in the medial or lateral rostral medulla. In sham-lesioned animals that were given paired presentations of noise and shock, subsequent presentation of the auditory stimulus caused a significant transient elevation of $A B P$ and time-dependent inhibition of the tail flick reflex evoked by radiant heat. Lesions of either the dorsal or the ventral PAG blocked the antinociceptive CR but did not significantly affect ABP responses. Lesions of the ventromedial, but not the lateral, rostral medulla blocked hypoalgesia. Rostral medullary lesions did not reliably affect stimulus-evoked cardiovascular responses or baseline ABP. These results indicate that antinociceptive and cardiovascular conditional responses are anatomically dissociable and support our proposal that conditional hypoalgesia is mediated by a serial neural circuit that includes the amygdala, PAG, and rostral ventromedial medulla.

IKey words: analgesia, blood pressure, periaqueductal gray, central gray, rostral medulla, defensive behavior, fear, Pavlovian conditioning, learning, memory]

When an animal is presented with an innocuous auditory signal for the occurrence of a noxious or stressful event, a series of integrated behavioral and physiological changes may be observed. Exposing a rat to an auditory stimulus that signals electric foot shock, for example, will result in gross somatomotor defensive responses such as freezing behavior, elevations in heart rate and arterial blood pressure (ABP), and an inhibition of nociceptive withdrawal reflexes or hypoalgesia (Iwata and

\footnotetext{
Received Nov. 2, 1993; revised Apr. 7, 1994; accepted May 19, 1994.

This research was supported by a grant-in-aid of neurobiology from the Whitehall Foundation and by NIH Grants DA07719 and MH49819.

Correspondence should be addressed to Fred J. Helmstetter, Department of Psychology, Garland Hall, University of Wisconsin, P.O. Box 413, Milwaukee, WI 53201.

Copyright @ 1994 Society for Neuroscience $0270-6474 / 94 / 147099-10 \$ 05.00 / 0$
}

LeDoux, 1988; LeDoux et al., 1988; Helmstetter and Bellgowan, 1993). The stimulus, which prior to being paired with shock will not produce such changes in behavior, gains control of these various response systems through the process of aversive Pavlovian conditioning. Presumably the expression of these fearrelated responses reflects the activation of brain systems that have evolved to subserve defensive functions (Adams, 1979; Blanchard and Blanchard, 1988; Fanselow, 1991).

Significant progress has recently been made in understanding the neural systems through which responses to fear-provoking stimuli are acquired and expressed. In the rat, the amygdala is currently considered an important forebrain region involved in several forms of aversive learning. The central nucleus of the amygdala projects to the periaqueductal gray (PAG) and other brainstem targets that appear to be important for the expression of the individual components of an integrated fear response (Ledoux et al., 1988; Davis, 1991; Shipley et al., 1991). Destruction of the central nucleus in the rat results in deficits in the behavioral as well as the autonomic expression of conditional fear, and chemical stimulation of the amygdala can result in behavioral and physiological responses that often resemble the animal's normal reaction to a signal for shock (Iwata et al., 1987; Helmstetter, 1992; Helmstetter et al., 1993). Conditional hypoalgesia (CHA), the activation of endogenous antinociceptive systems by a Pavlovian signal for shock, is also blocked by lesions of the amygdala (Helmstetter, 1992; Helmstetter and Bellgowan, 1993).

Hypoalgesia in response to a Pavlovian conditional stimulus (CS) appears to be expressed primarily through the brainstem antinociceptive circuit described by Basbaum and Fields (1984) in which neuronal activity in the periaqueductal gray (PAG) can inhibit inputs from primary afferent nociceptors via connections with cells in the medial region of the rostral ventral medulla (RVM) that project to the spinal cord dorsal horn. Lesions of the PAG block the conditional inhibition of radiant heat tail flick (TF) latency that rats show following exposure to an apparatus in which they had previously received foot shock (Kinscheck et al., 1984) and microinjection of the opioid antagonist naltrexone into the ventrolateral PAG blocks CHA in response to similar stimuli (Helmstetter and Landiera-Fernandez, 1990). There is also some evidence to indicate that lesions of the rostral medulla or dorsolateral funiculus will block either the acquisition or the expression of CHA (Watkins et al., 1982, 1983).

The brainstem regions involved in the performance of $\mathrm{CHA}$ appear to overlap extensively with those involved in other aversive or defensive responses. The PAG in particular appears to be responsible for the elaboration of multiple behavioral and 
physiological responses related to anxiety, fear, and defense. Lesions of the PAG can disrupt the normal expression of defensive topographies such as freezing behavior to species-typical danger stimuli or to a CS for shock (Liebman et al., 1971; LeDoux et al., 1988; Fanselow, 1991), and electrical or chemical stimulation of the PAG can provoke integrated patterns of aversive responses including behavioral and cardiorespiratory reactions along with hypoalgesia (Fardin et al., 1984; Bandler, 1988; Besson et al., 1991; Lovick, 1991; Depaulis et al., 1992). Studies directly addressing the relationship between antinociceptive and cardiovascular function in the PAG support the idea that these functions may be mediated by cells located in closely related regions within this structurc. In ancsthetized animals, chemical stimulation of the dorsal PAG will result in the simultaneous inhibition of the TF reflex and a marked pressor response while stimulation of the ventral PAG also results in antinociception but tends to produce decreases, rather than increases, in arterial blood pressure (Lovick, 1985, 1991; Sandkühler et al., 1991). However, in behaving animals large lesions of the PAG that are effective in disrupting the performance of freezing, a behavioral response that has repeatedly been shown to covary directly with hypoalgesia (e.g., Helmstetter and Fanselow, 1987), do not affect the transient elevations of ABP seen in animals presented with a tone that had been paired with shock (LeDoux et al., 1988). Both the antinociceptive and cardioregulatory functions of at least some PAG neurons may be ultimately expressed through connections with the rostral medulla (Lovick, 1985, 1991; Morgan et al., 1992; Verberne and Guycnet, 1992).

An accurate description of the essential neural circuits responsible for the expression of antinociceptive and cardiovascular conditional responses may be complicated by the fact that peripheral alterations in blood pressure can produce hypoalgesia (Randich and Maxiner, 1986; Watkins et al., 1990; Morgan and Fields, 1993). This hypoalgesia appears, at least in some cases, to be mediated by feedback from vagal afferants and involves a direct connection between the nucleus of the solitary tract and the RVM (see Randich and Gebhart, 1992, for a review). In a recent study Thurston and Randich (1992) showed that the spontaneous activity of populations of neurons in the RVM that are known to be directly involved in the modulation of the TF reflex (Fields et al., 1983) was also related to spontaneous changes in ABP. Stressful environmental stimuli often produce hypoalgesia and elevations in ABP simultaneously and it is possible that this stress-related inhibition of nociceptive reflexes occurs as an indirect or secondary consequence of transient hypertension. Therefore, since the presentation of an auditory CS that has been paired with shock will simultaneously cause a transient elevation in ABP (Iwata and LeDoux, 1988; LeDoux et al., 1988) as well as inhibition of the TF reflex (Helmstetter and Bellgowan, 1993), it is possible that CHA is at least partially a product of vagal afferent activation rather than the direct result of the operation of an independent descending serial neural circuit that includes the amygdala, PAG, and RVM.

The majority of studies that have addressed the involvement of the PAG and RVM in the expression of hypoalgesia and blood pressure regulation have relied on responses elicited by chemical or electrical stimulation in anesthetized rats. While a great deal of important progress has been made with this approach, the conditions under which these responses are measured are likely to be significantly different from those occurring in the intact behaving animal following exposure to the appro- priate environmental stimuli. Pavlovian conditioning techniques afford a well-controlled means by which endogenously generated elevations in blood pressure or hypoalgesia may be produced. The present study was designed to assess the involvement of the PAG and RVM in the simultaneous expression of cardiovascular and antinociceptive aversive conditional responses in the rat.

\section{Materials and Methods}

Animals, surgery, and histology. Subjects consisted of adult male LongFvans rats (Harlan Sprague Dawley, Madison, WI) weighing between 325 and $400 \mathrm{gm}$ at the time of testing. Animals were housed individually in hanging stainless steel cages with unlimited access to lab chow and water. The animal room was maintained on a $14: 10 \mathrm{hr}$ light/dark cycle. All experimental manipulations were performed during the light portion of the cycle. Animals were adapted to handling and transportation for several days prior to the beginning of the experiment. Because the objective of the study was to assess the contribution of selected brain areas to the expression rather than to the acquisition of conditional fear, all subjects were first given behavioral training as described below followed by lesions and subsequent recovery prior to behavioral testing for fearrelated changes in nociception and blood pressure.

Prior to surgery all animals were anesthetized with ketamine $\mathrm{HCl}$ (Ketaset; $100 / \mathrm{mg} / \mathrm{ml} / \mathrm{kg}$, i.p.) and sodium pentobarbital (Nembutal; 1.5 $\mathrm{mg} / \mathrm{rat}$, i.p.). Fach rat was implanted with a chronic indwelling femoral arterial catheter. The catheter was constructed from a $4 \mathrm{~cm}$ piece of polyvinyl tubing $(0.5 \mathrm{~mm}$ i.d., $0.8 \mathrm{~mm}$ o.d.; Dural Plastics, Dural, NWT, Australia) connected to Tygon microbore tubing ( 0.02 inch i.d., 0.06 inch o.d.) by 22 gauge stainless steel hypodermic tubing. The polyvinyl end of the catheter was carefully inserted into the right femoral artery and anchored to the surrounding muscle tissue with nylon sutures. The remainder of the Tygon tubing was threaded subcutaneously and externalized at the back of the neck. Patency of the catheter was maintained using a $5 \%$ heparin-saline solution.

Immediately following catheter implantation, each subject received electrolytic lesions of one of four target structures or a sham lesion control treatment. Lesion electrodes were constructed from stainless steel insect pins $(00$ gauge $)$ that were insulated except for approximately $0.5 \mathrm{~mm}$ at the tip. Electrodes were stereotaxically positioned in the target structure and a constant current DC lesion maker (Grass Instruments Inc.) was used to pass a $0.1 \mathrm{~mA} \mathrm{DC}$ current for $45 \mathrm{sec}$.

Initial stereotaxic coordinates were derived from the atlas of Paxinos and Watson (1986). Animals in the medial rostroventral medulla group (medRVM; $N=9$ ) received a single lesion centered on the midline in the ventromedial medulla (AP -11.0, ML 0, DV -10.6). The lateral rostroventral medulla group (latRVM; $N=10$ ) received bilateral lesions on each side of the target area for the medial group (AP -11.8, ML \pm 2.4 , DV -10.6 ). These larger lesions were included to control for nonspecific damage to the rostral medulla and were not intended to destroy ventrolateral cell groups involved in the modulation of ABP. Rats in the ventral periaqueductal gray group (vPAG; $N=7$ ) received a single lesion centered ventral to the aqueduct in the central gray (AP $-7.6, \mathrm{MI},+0.3, \mathrm{DV}-6.8$ ). The final lesion group (dPAG; $N=9$ ) received bilateral lesions in the dorsolateral/dorsomedial area of the PAG (AP -7.6, ML \pm 1.0 , DV -5.3). For each lesion site separate groups of control animals (SHAM) were treated identically to lesioned subjects except that the electrodes were lowered into each of the target structures but no current was passed $(N=31)$.

The rats were allowed to recover from surgery for $5 \mathrm{~d}$ before being tested. Any animal that did not fully recover in that period of time (as indicated by lethargy or extreme weight loss) or was found to have had an inoperable or occluded catheter did not continue in the study. One animal each in the dPAG, medRVM, and SHAM groups and two animals in the vPAG group failed to recover completely from surgery. Animals with inoperable blood pressure catheters at the time of testing were eliminated from the dPAG $(N=2)$, medRVM $(N=1)$, latRVM $(N=3)$, and SHAM $(N=3)$ groups. Thus, a total of 14 animals that were prepared were not tested.

After testing, all rats were euthanized with an overdose of sodium pentobarbital/ethanol solution and perfused transcardially with $10 \%$ buffered formalin. The brains were removed and stored overnight in a $20 \%$ sucrose-buffered formalin solution. Frozen $40 \mu \mathrm{m}$ sections were mounted on slides and stained with cresyl violet. Lesions were reconstructed with the aid of a rat brain atlas (Paxinos and Watson, 1986). 
Apparatus. Four standard rat observation chambers were used during fear conditioning. Each chamber was constructed from Plexiglas and stainless steel and measured $23.5 \times 29 \times 19 \mathrm{~cm}$. White noise stimuli ( $74 \mathrm{~dB}$, A scale) were presented via a speaker $(9 \times 9 \mathrm{~cm})$ mounted on a wall of each chamber. The floor of each observation chamber was constructed from stainless steel rods spaced approximately $1.25 \mathrm{~cm}$ apart through which AC scrambled foot shock could be delivered. Each chamber was connected to its own shock generator/scrambler (GrasonStadler) and located within a sound- and light-attenuating chest equipped with ventilation fans that provided constant background noise at 68$70 \mathrm{~dB}$.

Tail flick testing and measurement of $\mathrm{ABP}$ were conducted in a separate room using restraining tubes constructed from PVC pipe $(7.6 \times$ $18 \mathrm{~cm}$ ) with clear Plexiglas attached to one end. Small holes were drilled into the front and sides of the tubes to provide adequate ventilation. TF latencies were measured using a custom made apparatus that consisted of a steel box containing a $500 \mathrm{~W}$ light bulb that delivered radiant heat to the distal third of the rat's tail through a $3 \mathrm{~mm}$ aperture located directly above the bulb. A photocell located $9 \mathrm{~cm}$ above the aperture was positioned such that lateral movement of the tail after activation of the heat source terminated the trial. An integrated digital timer recorded the animal's response latency to within $10 \mathrm{msec}$. In order to prevent tissue damage, failure to respond within $15 \mathrm{sec}$ of heat onset resulted in the automatic termination of the trial and assignment of a latency of $15 \mathrm{sec}$.

Arterial blood pressure was simultaneously measured using a Statham P23AA pressure transducer. The output of the transducer was amplified with a DC preamplifier (Grass Instruments Inc.) and routed to an A/D converter and microcomputer. The computer recorded ABP and controlled the presentation of all stimuli and the activation of the TF apparatus. Transducer output was continuously stored throughout the test session for later off-line analysis. All statistical analyses were conducted on 1 sec samples of mean arterial pressure (MAP).

Training procedure. Prior to surgery subjects were exposed to an aversive classical conditioning procedure on each of three consecutive days. Each rat received a single $40 \mathrm{~min}$ session per day in which they were exposed to a total of 10 presentations of white noise $(74 \mathrm{~dB} / 60$ sec) and scrambled AC foot shock $(2.0 \mathrm{~mA} / 1.0 \mathrm{sec})$ with an avcrage intertrial interval of 2 min. The majority of subjects received paired presentations of the noise and shock such that the shock always occurred at noise offset. A subset of of the sham-lesioned animals were assigned to a control group that received an equal number of auditory stimuli and shocks that were explicitly unpaired (i.e., noise and shock never occurred within 1 min if each other). On the second and third day of behavioral training rats were placed in the restraining tubes and left undisturbed in an isolated quiet room for $1 \mathrm{hr}$. Restraint adaptation was conducted approximately $5 \mathrm{hr}$ after training with noise and shock. After the completion of fear conditioning and restraint adaptation all animals were prepared with catheters and electrolytic lesions as described above.

Testing procedure. After at least $5 \mathrm{~d}$ recovery from surgery each rat was removed from its home cage and transportcd to an isolated testing room. The rat was placed in a restraining tube and the end of its catheter was threaded through an opening in the tube. The catheter was connected to the pressure transducer in preparation for ABP recording and the animal was left restrained and undisturbed for $10 \mathrm{~min}$ before any data were recorded.

Baseline ABP was then collected for $10 \mathrm{~min}$ before any TF testing or presentation of auditory stimuli. The test session consisted of a total of $15 \mathrm{TF}$ trials with a constant 2 min interval between trials. The first four trials were conducted in the absence of any auditory stimuli and were used as an index of baseline nociception for each animal. A $60 \mathrm{sec}$ presentation of the white noise $\mathrm{CS}(74 \mathrm{~dB}$, as measured in the restraining tube at the position of the animals head) occurred on TF trials 5,8 , and 13. The noise stimulus was activated $45 \mathrm{sec}$ prior to onset of radiant heat so that TF latency was recorded during the final $15 \mathrm{sec}$ of the white noise. Thus, stimulus-related elevation in ABP and inhibition of the TF reflex were assessed at three separate points within the session.

Data analysis. Tail flick latencies are expressed as means and standard error of the means of all subjects included within each group. To minimize the effects of individual differences in resting MAP, all stimulusevoked blood pressure responses were converted to reflect the relative amount of change from prestimulus baselines by subtracting the mean of a $15 \mathrm{sec}$ period prior to CS onset from each $1 \mathrm{sec}$ MAP sample recorded during presentation of the CS. Since the responses of individual
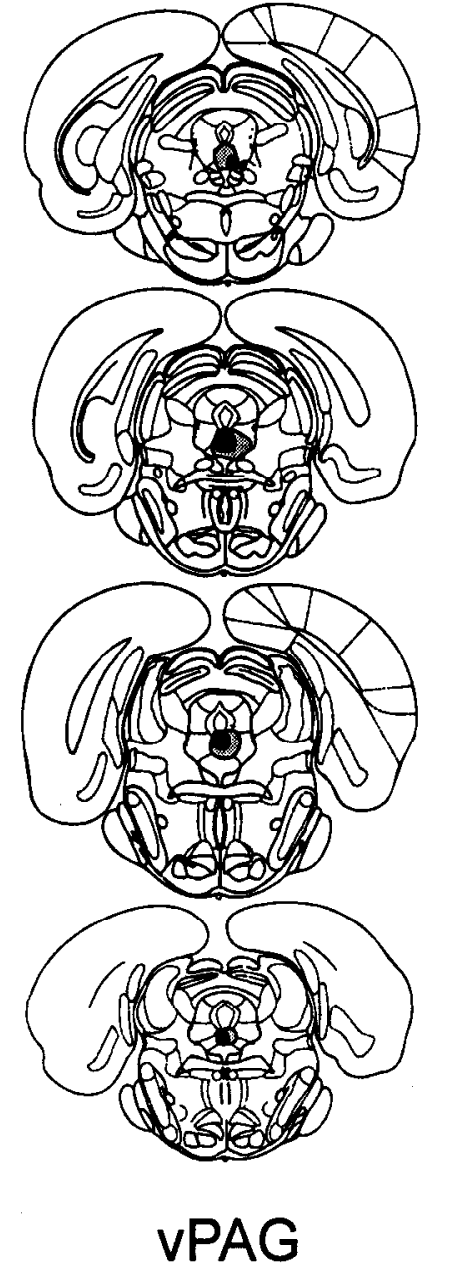
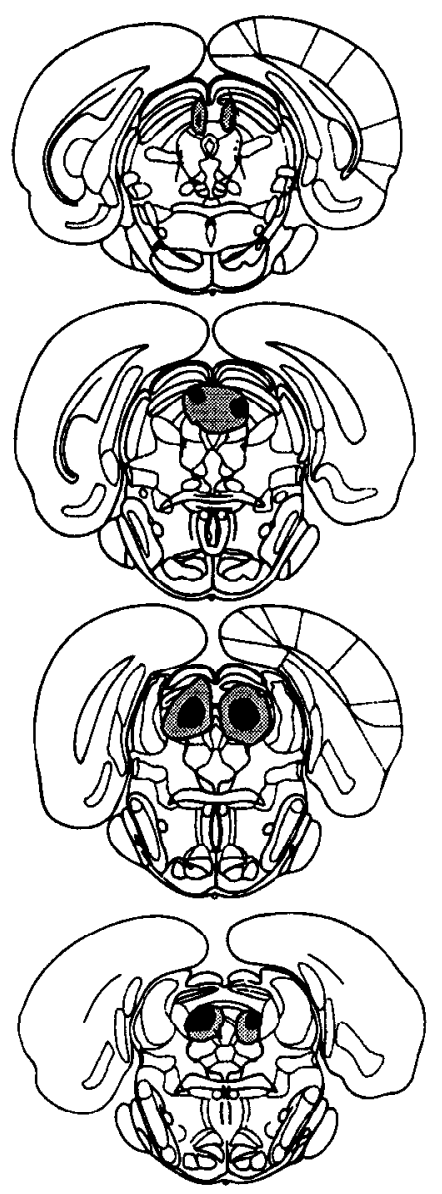

dPAG
Figure 1. Examples of typical large and small lesions of the ventral (left) and dorsal (right) periaqueductal gray. Ventral lesions were normally centered on the midline and destroyed the dorsal raphe as well as portions of the ventrolateral PAG. Dorsal PAG lesions were made bilaterally extending ventrally to the aqueduct and dorsally into the superior colliculi.

sham-lesioned control groups were nearly identical, animals that had electrodes lowered into each of the target structures were pooled to form paired and unpaired sham-operated control groups. The reliability of overall differences between treatment groups was assessed using mixed model analysis of variance (ANOVA) on each dependent variable. Stimulus-evoked changes in TF latency were analyzed with a repeated measures ANOVA treating test trial (TF latency during and immediately following a given $\mathrm{CS}$ ) and trial block $\left(\mathrm{CS}_{1}-\mathrm{CS}_{3}\right)$ as within subject factors and group assignment as a between subject factor. Blood pressure responses recorded during the first $10 \mathrm{sec}$ of each CS presentation were analyzed in a similar manner by treating time (1-10 sec) and stimulus presentation $\left(\mathrm{CS}_{1}-\mathrm{CS}_{3}\right)$ as within subject factors and group as a between subjects factor. Predicted differences between individual pairs of group means were assessed by independent pairwise planned comparisons (Hays, 1988).

\section{Results}

\section{Histology}

All animals that were included in the vPAG lesion group had complete damage to the medial portion of the central gray veniral to the aqueduct and to the dorsal raphe nucleus $(N=5)$. Four of the five animals sustained extensive damage in the ventrolateral region of the PAG, as defined by Paxinos and Watson (1986) on one or both sides. The left panel of Figure 1 
Figure 2. Serial reconstructions of the largest and smallest lesions in the medial (left) and lateral (right) RVM groups. Medial lesions always included the $n$. raphe magnus and parts of the $n$. reticularis paragigantocellularis. Lateral lesions never overlapped with structures destroyed by the medial lesions. None of the animals in the lateral group evidenced damage to the $\mathrm{C} 1$ adrenergic cell area.

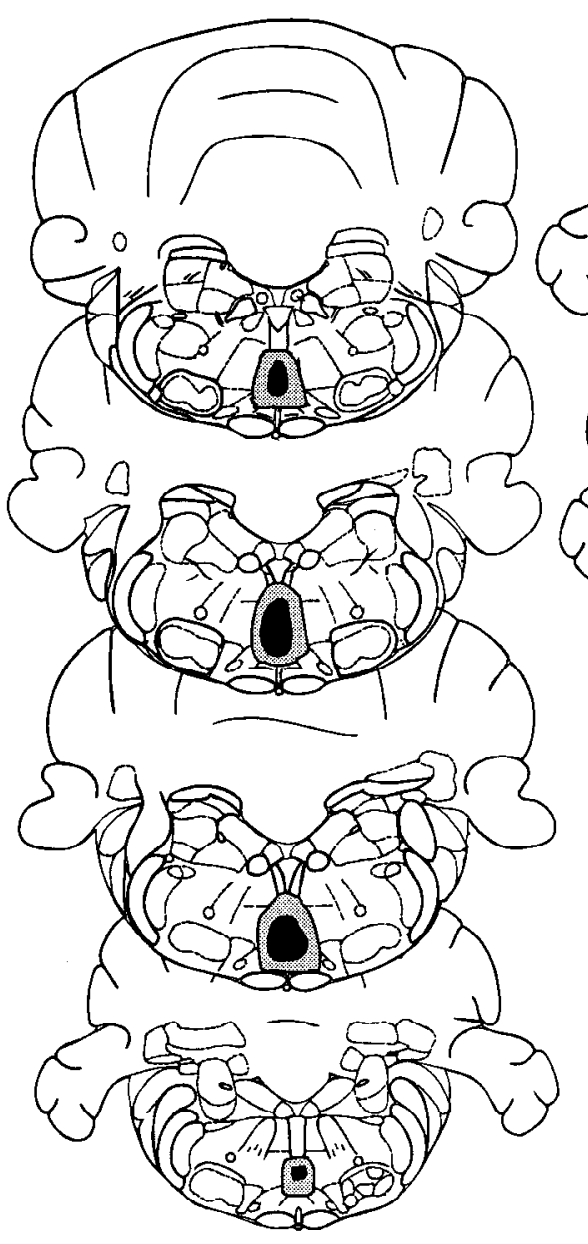

medRVM

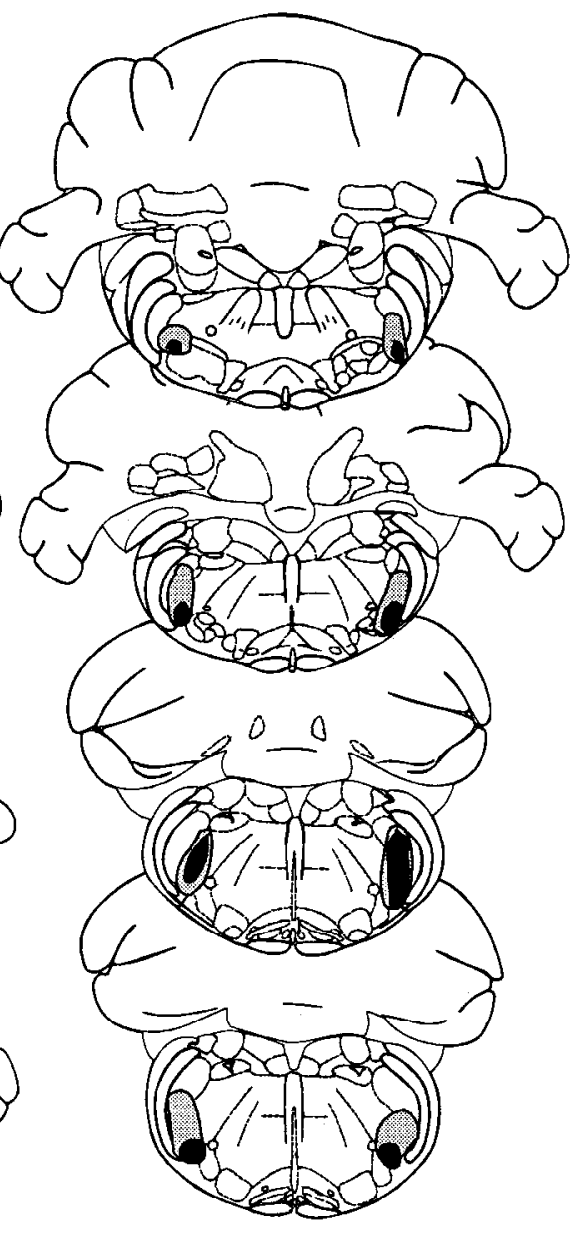

latRVM represents animals with typical large and small lesions included in the vPAG group.

All rats included in the dPAG lesion group had rather extensive damage to the dorsomedial and dorsolateral regions of the PAG $(N=6)$. In all cases there was some damage in the deep and intermediate layers of the superior colliculi bilaterally. None of the lesions extended significantly into the ventral or ventrolateral PAG. Examples of the largest and smallest dPAG lesions which were included in the analysis may be found in the right panel of Figure 1.

Only animals with extensive damage to all or part of the nucleus raphe magnus were included in the medRVM group ( $N$ $=7$ ). Within this group all individuals had lesions that also destroyed some portions of $n$. gigantocellularis and the most caudal part of the pontine reticular formation. Portions of the dorsal gigantocellular reticular nucleus and predorsal bundle were damaged in all but one animal whose lesion extended more ventrally to include portions of $n$. raphe pallidus and the pyramidal tract. The lesions in five of the seven animals extended caudally into the rostral extent of $n$. raphe obscurus.

The criteria for inclusion in the latRVM group were that the lesions be lateral to and not overlapping the areas destroyed in the medRVM lesion group. All animals included for analysis in the latRVM group had damage in the area of the parvocellular reticular nucleus, pars alpha $(N=7)$. In some cases the lesions extended into the trigeminal complex (three of seven), lateral reticular nuclei (three of seven), or facial nuclei (three of seven) bilaterally. None of the latRVM lesions extended ventrally into the $\mathrm{Cl}$ adrenergic cell area. One animal showed minimal unilateral damage to the nucleus of the solitary tract. Reconstructions of the largest and smallest lesions included in the medial and lateral RVM groups are shown in Figure 2.

\section{Antinociception}

The mean of three radiant heat TF trials conducted prior to the presentation of any auditory stimuli was used as an index of each subject's baseline nociceptive reactivity. A comparison of the mean pre-CS TF latencies from all treatment groups indicated that neither type of training (paired vs unpaired noise and shock) nor lesion had a significant impact on tail flick latencies prior to CS presentation $[F(5,46)=2.295, p>0.05]$ (see leftmost point on Fig. 3).

CHA in response to shock-associated white noise is indicated by the inhibition of TF reflexes seen in sham-operated animals that were exposed to paired presentations of noise and shock (Fig. 3). The initial overall ANOVA on TF data indicated that significant changes in latency occurred as a function of group assignment over the three blocks of TF trials associated with individual CS presentations as indicated by the group $\times$ block interaction $[F(10,92)=2.757, p<0.005]$, and over the three 
TF trials within each block as indicated by the group $\times$ trial interaction $[F(10,92)=3.193, p<0.001]$. For most groups, $\mathrm{TF}$ latency tended to decrease within blocks of trials. A reliable trial $\times$ block interaction $[F(4,184)=7.931, p<0.001]$ indicated that on average the rate at which animals approached baseline levels of TF latency following exposure to the CS differed across the three stimulus presentations. The three-way group $\times$ block $\times$ trial interaction was not statistically significant $[F(20,184)$ $=1.058, p>0.05]$.

Comparison of individual group mean TF latencies measured during the first $60 \mathrm{sec} C S$ presentation $\left(\mathrm{CS}_{1}\right)$ revealed that rats that had received paired training with noise and shock showed significantly longer latencies than did the unpaired controls $[F(1$, $46)=26.64, p<0.001]$. Significant TF inhibition was still evident at the second TF trial conducted 2 min after the CS presentation $[F(1,46)=4.351, p<0.043]$, but not at $4 \mathrm{~min}$ after the stimulus. The paired control group continued to show an increase in TF latency upon presentation of $\mathrm{CS}_{2}[F(1,46)=$ $9.175, p<0.004]$, which persisted for $2 \mathrm{~min}$ after $\mathrm{CS}$ offset $[F(1,46)=5.171, p<0.028]$. There were no significant differences in TF latency between any of the groups during or after the third CS presentation.

Lesions of the dorsal or ventral regions of the PAG had similar effects on the expression of CHA. As can be seen in Figure 3, CS-evoked inhibition of the TF reflex was significantly attenuated relative to paired sham-operated controls in animals with lesions of either the ventral $[F(1,46)=10.236, p<0.002]$ or the dorsal PAG $[F(1,46)=18.573, p<0.001]$. Indeed, mean TF latencies for these groups were similar to those of intact rats that had received unpaired presentations of the noise and shock prior to surgery. During the second CS presentation, stimulusevoked inhibition of TF latencies was still attenuated in the dorsal PAG lesion group $[F(1,46)=4.084, p<0.05]$. While the response of animals in the ventral PAG lesion group was similar to that of the dorsal lesioned animals, the difference between this group and paired sham-operated controls was statistically marginal $[F(1,46)=2.939, p<0.093]$. As stated above, no two groups differed significantly during or after the presentation of $\mathrm{CS}_{3}$.

Figure 4 represents the effects of lesions of the rostral medulla on CHA during the test session. The data from pooled shamoperated groups that appears in Figure 3 are repeated to facilitate direct comparison to lesioned animals. As staled above, lesions were without effect on baseline TF responses recorded prior to the first CS presentation. Lesions of the medial RVM significantly attenuated the hypoalgesic response that occurred during presentation of $\mathrm{CS}_{1}$ relative to sham-operated controls. Even though they had received training identical to that of the paired sham group prior to surgery, animals with medRVM lesions responded in a manner similar to the unpaired controls. This attenuation of CHA in the medRVM group was seen consistently throughout the first $[F(1,46)-14.847, p<0.001]$ and second $[F(1,46)=7.676, p<0.008] \mathrm{CS}$ presentations as well as during post-CS periods. While significant $\mathrm{CHA}$ was not seen in control animals during the final CS presentation, the general relationship between the means of control and medRVM rats remained consistent. In contrast, lesions placed more laterally in the rostral medulla that destroyed approximately twice as much tissue on average as did the medial lesions did not significantly affect the performance of CHA during any of the CS presentations or post-CS periods. The latRVM group showed normal inhibition of TF during and immediately following $\mathrm{CS}_{1}$

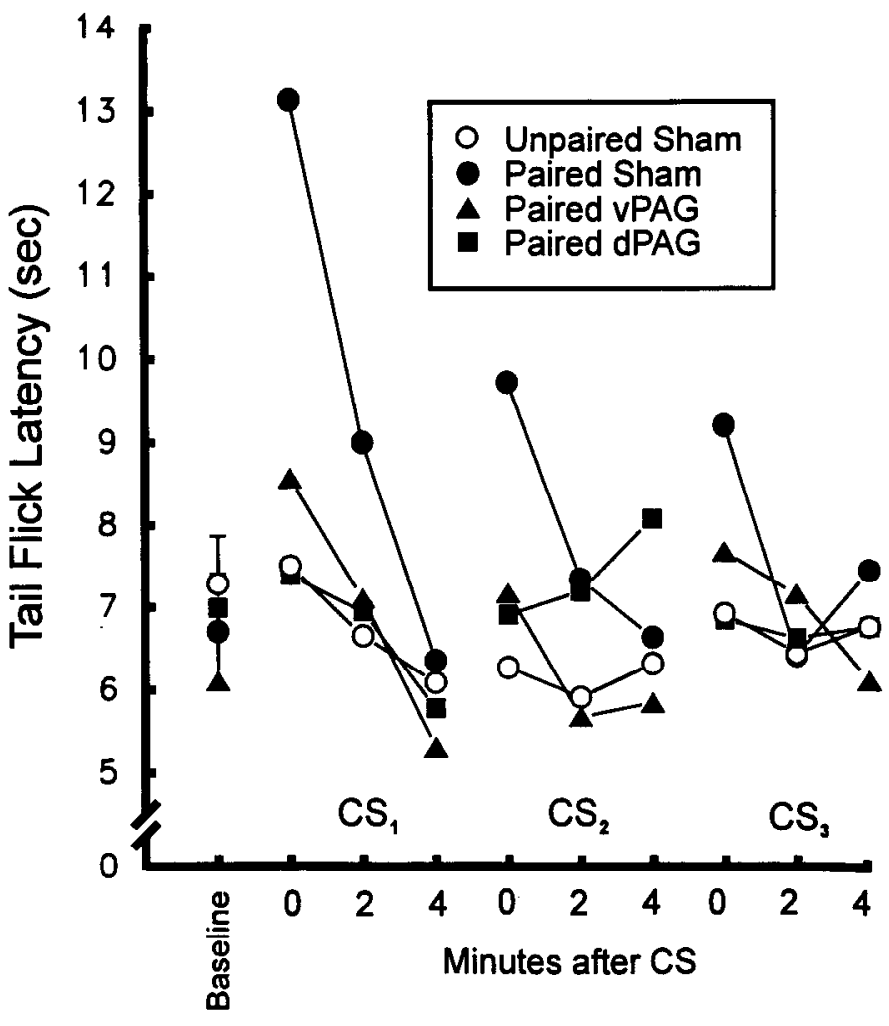

Figure 3. Mean TF latencies recorded before and after presentation the CS. The point labeled "baseline" represents the mean of three TF trials conducted at $2 \mathrm{~min}$ intervals. There were no baseline differences between groups of animals due to lesions or behavioral training condition. CS presentation caused a significant inhibition of the TF reflex during the first and second stimulus presentation in control animals that had been given paired training. Lesions of either the dorsal or the ventral PAG blocked this hypoalgesia.

$[F(1,46)=1.046, p>0.05]$ and $\operatorname{CS}_{2}[F(1,46)=1.945, p>$ 0.05 ] relative to the paired sham controls.

\section{Arterial blood pressure}

Baseline MAP values for each group measured over a $60 \mathrm{sec}$ period immediately prior to TF testing is shown in Figure 5. As was the case for baseline TF latencies, a one-way ANOVA indicated that there were no significant differences in baseline MAP between groups of rats due to type of training or lesions $[F(5,46)=1.244, p>0.05]$.

Exposure to the $\mathrm{CS}$ in paired control animals resulted in a transient increase in ABP primarily during the first $10 \mathrm{sec}$ of the CS period. Figure 6 represents raw ABP data collected from a single subject in the paired control group during the first CS presentation. An overall repeated-measures ANOVA on the mean change from baseline scores for each group over the first $10 \mathrm{sec}$ of each CS presentation produced a significant group $\times$ CS presentation $\times$ time interaction $[F(90,828)=1.826, p<$ $0.001]$, which supports the general observation that blood pressure responses differed as a function of treatment group with respect to both CS trial and time following CS onset. As can be seen in Figure 7, animals that received paired training with the CS and shock prior to the test session showed a much larger time-dependent elevation in MAP following CS onset than did the unpaired control group.

In general, the largest change from baseline MAP occurred between 3 and 4 sec after CS onset. Planned comparisons be- 


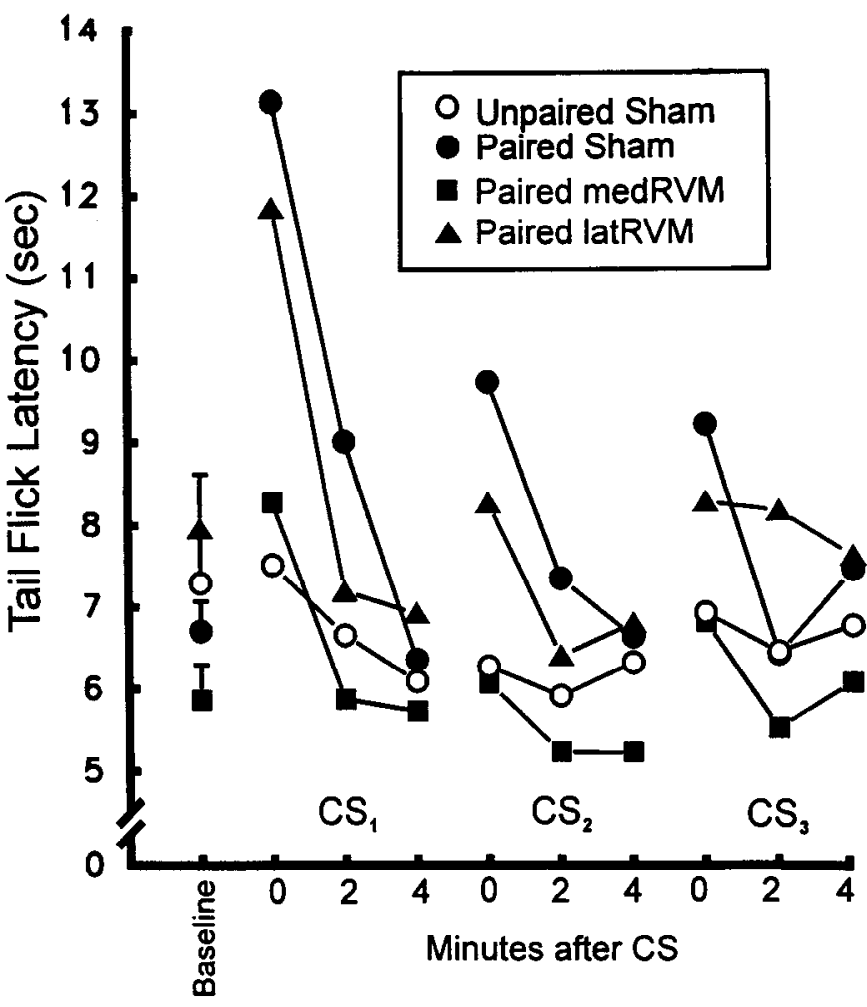

Figure 4. Lesions of the medial RVM did not affect baseline TF latencies but did significantly attenuate the antinociception seen during CS presentations. Control lesions in the lateral RVM did not block conditional hypoalgesia. Data from sham-operated controls are repeated from Figure 3 for comparison.

tween the means of the paired and unpaired sham controls indicated that animals that received paired training showed a significantly larger ABP response at $3 \mathrm{sec}$ after CS onset $[F(1$, $46)=11.249, p<0.001]$. A significant increase in paired versus unpaired animals was also detected in sham control animals 3 sec following the second CS presentation $[F(1,46)=9.431, p$ $<0.004]$, but as in the case of TF inhibition no significant differences were detected between any two groups of animals during the third CS presentation $[F(5,46)<1.0]$.

While antinociception was blocked by lesions of either the dorsal or ventral PAG, the results depicted in Figure 7 indicate that this was not the case for conditional ABP responses. All of the PAG lesioned animals showed a clear increase in ABP following presentation of the CS. Although it appears that vPAG lesioned animals reacted with an exaggerated pressor response to the first CS presentation, this increase in arterial pressure was not significantly greater at $3 \mathrm{sec}$ after CS onset than that of the sham-operated control group that received equivalent paired training $[F(1,46)<1.0]$. No reliable differences in peak ABP response were found between the paired sham and PAG lesion groups, or between PAG lesion groups, during any CS presentation $[F \mathrm{~s}(1,46)<1.0]$.

In contrast to hypoalgesia, the increase in $\mathrm{ABP}$ in response to $\mathrm{CS}_{1}$ was not affected by lesions of the medRVM. Figure 8 depicts the effects of medial and lateral medullary lesions on conditional stimulus-evoked pressor responses relative to the sham-operated control groups. Animals that received either medial or lateral RVM lesions and paired training showed an ABP response that was not significantly different from the paired control animals during the first and second CS presentation

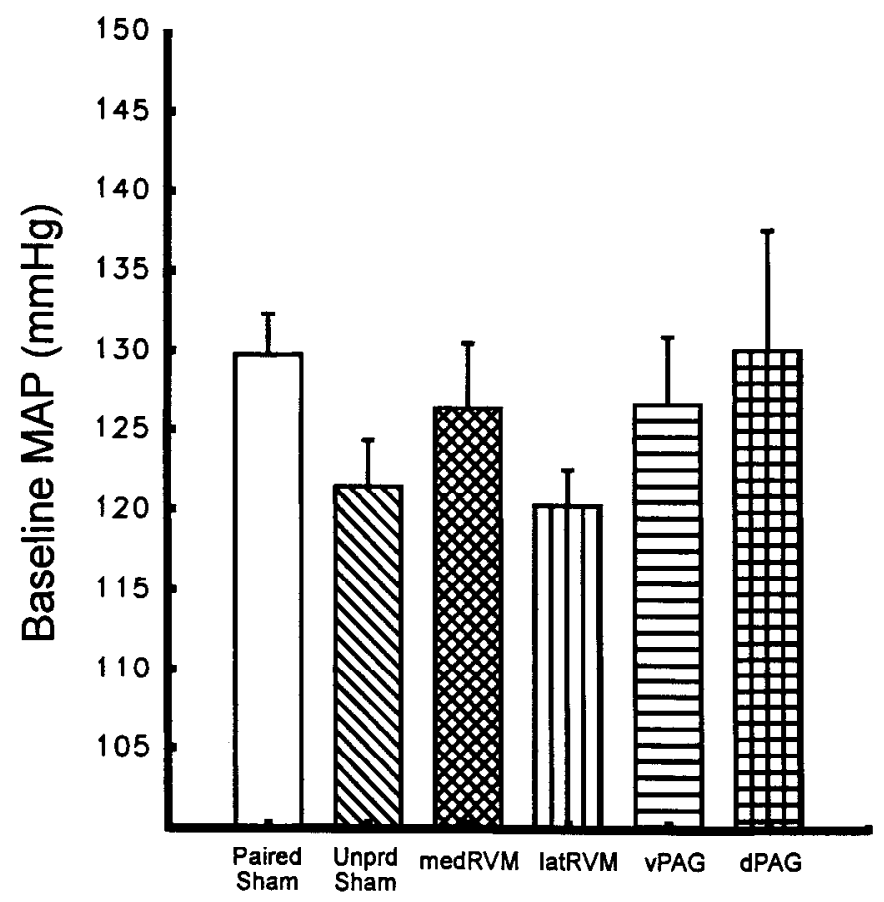

Figure 5. Baseline mean arterial pressure (MAP) recorded over a 60 period during the test session prior to any tail flick testing. There were no significant differences in resting MAP between any two groups of rats.

$[F \mathrm{~s}(1,46)<1.0]$. While the means of the two lesion groups were slightly higher than those of controls during the initial half of the final noise presentation, analysis indicated that there were no reliable differences between any two groups during this stimulus period.

\section{Discussion}

The results of this study indicate that while exposure to an innocuous auditory stimulus that has served as a signal for shock can simultaneously result in both hypoalgesia and a transient increase in ABP, the neural circuits responsible for the expression of these responses appear to be somewhat independent. As we have argued previously, the expression of CHA to an auditory CS most likely relies on a serial descending circuit that includes the basolateral and central regions of the amygdala, the ventrolateral PAG and the rostral ventromedial medulla. Moderately sized electrolytic lesions of the PAG and medial RVM made after fear conditioning significantly attenuated the performance of CHA without affecting baseline TF latencies, resting $\mathrm{ABP}$, or cardiovascular CRs. Several aspects of the present results have important implications for understanding the brain mechanisms of defensive behavior, aversive Pavlovian conditioning, and environmentally elicited hypoalgesia.

Nonlesioned animals that had previously received paired presentations of the CS and shock in an observation chamber showed highly reliable simultaneous increases in TF response latency and ABP during subsequent nonreinforced presentations of the CS. Animals that were treated identically except that the two stimuli were not paired during training showed minimal changes in either variable as a function of CS presentation. Furthermore, as the CS was repeatedly presented in the absence of shock during the test session, both responses showed reliable extinction. It is interesting to note that while the CS was always pre- 

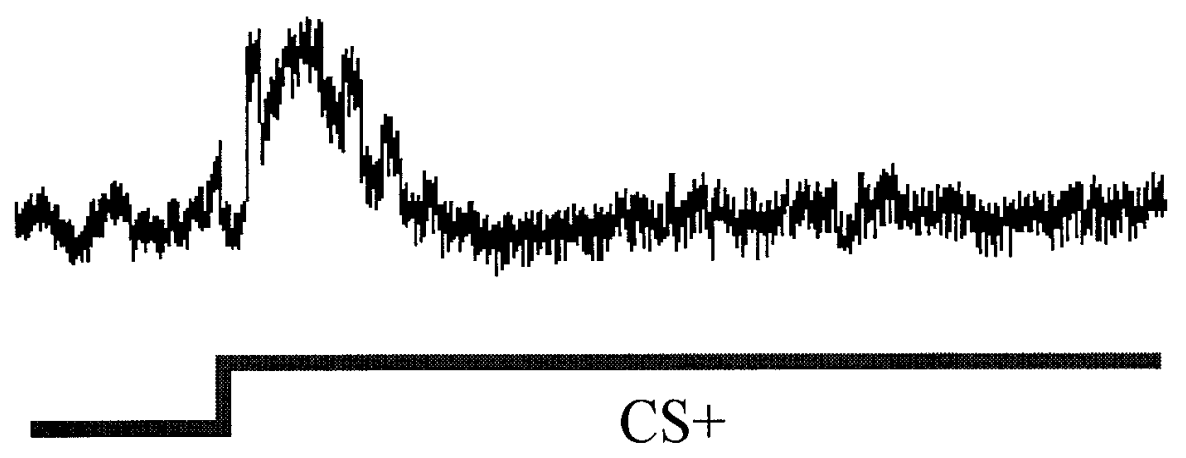

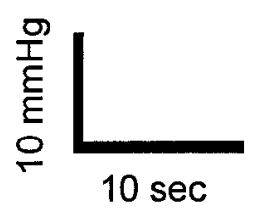

Figure 6. Example of an arterial blood pressure CR in response to the presentation of an auditory signal that had previously been paired with foot shock. This response was recorded in an a control animal during the first CS presentation of the test session. sented for $60 \mathrm{sec}$, the maximal ABP response always occurred during the first 5-10 sec after stimulus onset (Fig. 6; see also Iwata and LeDoux, 1988; LeDoux et al., 1988). A series of studies recently completed in our laboratory indicate that maximal inhibition of the TF reflex in this paradigm may also occur during the first $15 \mathrm{sec}$ of a $60 \mathrm{sec}$ auditory CS (Helmstetter and Gale, unpublished observations). These similarities in CR topography between blood pressure and CHA support the idea that the performance of these responses share common neural substrates at some level (i.e., the central nucleus of the amygdala).

There is a clear relationship between experimentally induced hypertension and antinociception in rats (Randich and Maxner, 1986; Watkins et al., 1990; Randich and Gebhart, 1992; Morgan and Fields, 1993). Elevation of ABP has been shown to result in inhibition of the TF reflex and this effect may be at least partially mediated by vagal afferent feedback and descending control from the rostral medulla. Reciprocal projections between the nucleus of the solitary tract and the RVM exist and studies in anesthetized rats have shown that antinociception following electrical stimulation of vagal afferants is blocked by temporary inactivation of the RVM. Furthermore, the activity of cells located in the RVM that presumably are directly responsible for modulation of the TF reflex (Fields et al., 1983) is also related to changes in ABP (Thurston and Randich, 1992; Morgan and Fields, 1993).

However, some of the available evidence does not support the idea that peripheral alterations in blood pressure directly result in TF inhibition mediated by the RVM. Morgan and Fields (1993) reported that although there was a reliable relationship between the activity of RVM on- and off-cells and increases in ABP produced by volume expansion following intravenous administration of Ficoll, reversible inactivation of RVM neurons with lidocaine had no effect on Ficoll-induced

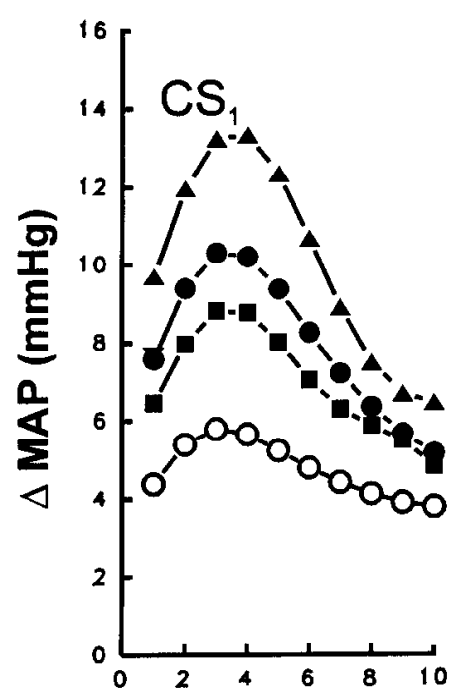

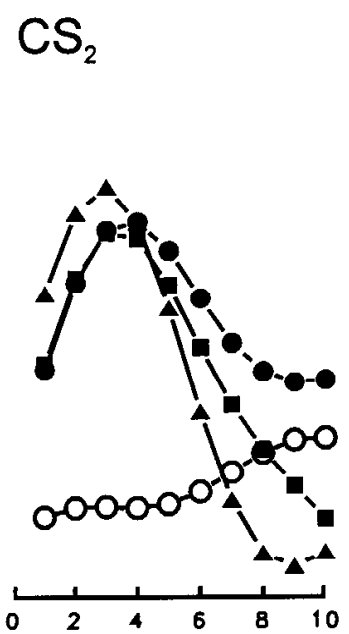

$\mathrm{CS}_{3}$

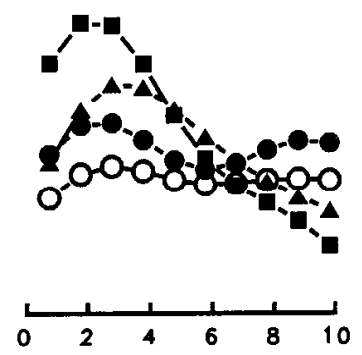

Seconds after CS onset
A Paired VPAG
Figure 7. Presentation of the CS resulted in a significant elevation of $\mathrm{ABP}$ in all animals given paired training with noise and shock. Lesions of the PAG did not significantly affect the performance of this response. Reliable elevations in ABP could not be detected during the third CS presentation in the session. Each point represents $1 \mathrm{sec}$ of mean arterial pressure recorded during the initial $10 \mathrm{sec}$ of the noise. 
Figure 8. Blood pressure CRs were not affected by lesions of the medial or lateral RVM. Lesion groups did not differ from paired controls during the first and second CS presentations. No significant differences existed between any two groups during the third CS period.

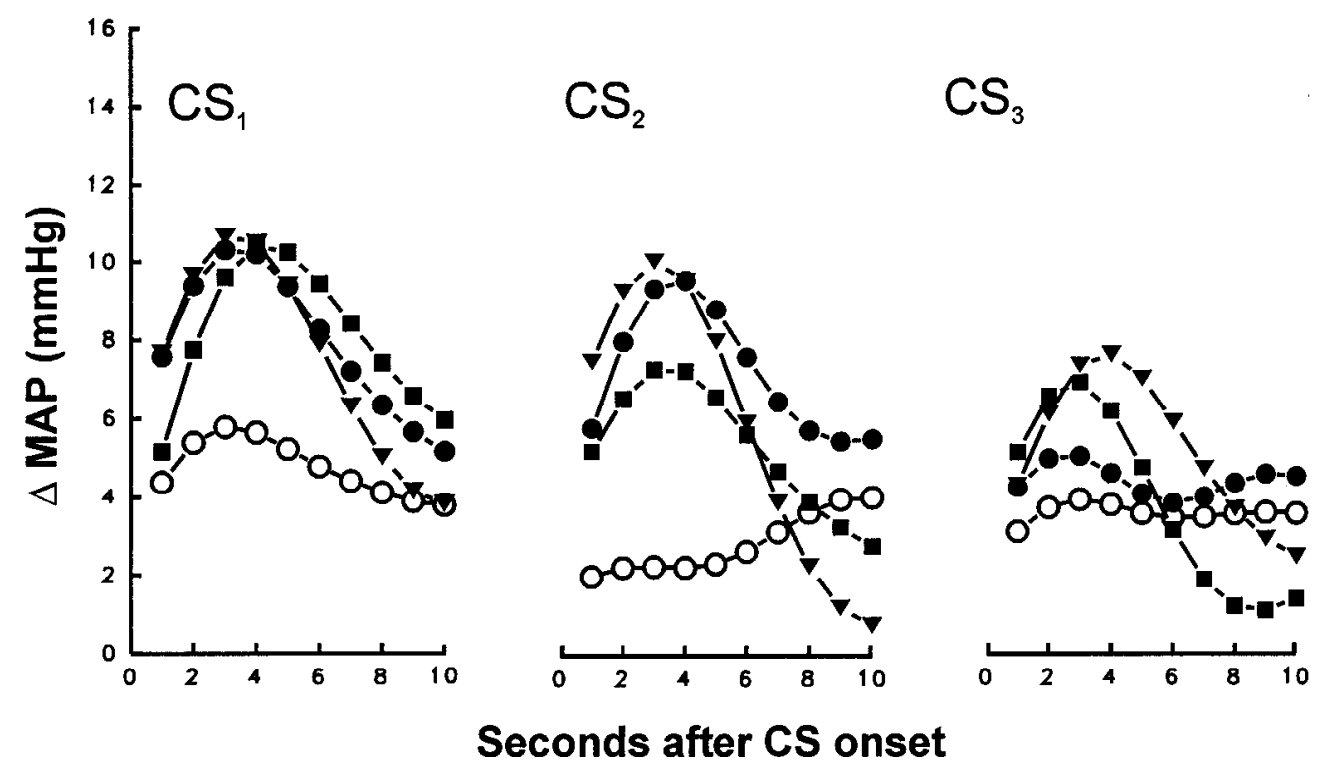

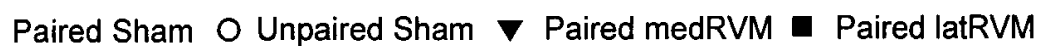

TF inhibition. Based on these results, Morgan and Fields (1993) concluded that vagal afferent stimulation can affect the activity of RVM cells but produces hypoalgesia via some other mechanism. It is interesting to also note that TF inhibition following intravenous administration of low doses of opioid receptor agonists is sometimes, but not always, blocked by inactivation of the RVM (see Randich and Gebhart, 1992). In the present study, lesions that were intended to destroy a large number of nociceptive modulatory neurons in the medial RVM blocked TF inhibition in response to a signal for shock but did not affect conditional changes in arterial pressure. These results indicate that while the activity patterns of cells in this region of the RVM may relate to changes in both nociception and ABP, these cells do not participate in producing the dynamic changes in $A B P$ seen following exposure to the CS. The present results, taken together with those of Morgan and Fields (1993), indicate that if peripheral elevations in $\mathrm{ABP}$ inhibit the TF reflex via some non-RVM-dependent mechanism, the transient changes in ABP seen in the present study in all subjects, including those with lesions of the RVM, must not be large enough to trigger this alternative antinociceptive mechanism. It is also quite possible that the relationship between modulation of ABP and hypoalgesia may be substantially different in the awake behaving animal from that normally seen in anesthetized preparations.

Relatively small electrolytic lesions of either the dorsal or the ventral PAG were effective in selectively blocking CHA without changing baseline nociceptive reactivity. The same lesions had no effect on conditional ABP responses to the same stimulus. This lack of effect on cardiovascular CRs is consistent with the results of LeDoux et al. (1988), who reported that neither electrolytic nor ibotenic acid lesions of the PAG affected the performance of ABP CRs to a tone that had been paired with shock. The same lesions were effective in attenuating the somatomotor freezing response to the same stimulus. In contrast, lesions made in the lateral hypothalamus did not affect freezing but did attenuate the cardiovascular CR. LeDoux et al. (1988) concluded that different efferent projections from the central nucleus of the amygdala are responsible for the expression of these two responses. When these findings are taken together with the present results, it appears that the neural substrates for CHA are more closely related to those of defensive freezing bchavior than to the cardiovascular components of conditional fear.

Our failure to find an effect of either dorsal or ventral PAG lesions on conditional ABP responses would not be predicted based on existing data gathered using direct chemical or electrical stimulation of this structure in anesthetized animals. For example, Lovick (1991) has shown that stimulation of the rat PAG results in inhibition of the TF reflex that is accompanied by highly reproducible stimulation-related changes in respiration, vascular conductance and ABP. Specifically, dorsal PAG stimulation results in a pressor response while ventral stimulation causes a transient reduction in ABP. Is it believed that both types of $\mathrm{ABP}$ alterations are mediated by connections between the PAG and cardioregulatory neurons in the rostral ventrolateral medulla. Lovick (1991) interprets these effects in the context of the PAG's often discussed role of integrating the multiple components of normal defensive responses. If the PAG plays an essential general role in stress-related cardiovascular adjustments in behaving animals then lesions of this structure should clearly have some impact on fear-related elevations in ABP. It is of course possible that the PAG instead plays a more restricted role in these processes and the nature or amount of stress experienced by the animals in this study was incompatible or insufficient in quantity to directly engage regulatory mechanisms within this structure.

Several studies have indicated that the relationship between hypoalgesia and other defensive behaviors may be different at different regions within the PAG. Electrical stimulation of either the dorsal or the ventral PAG in awake freely moving animals results in hypoalgesia, but the gross behavioral reactions of the animals differ significantly with dorsal stimulation causing increased activity and "aversive" behavior patterns that are not seen during or after ventral PAG stimulation (Cannon et al., 1982; Fardin et al., 1984; Besson et al., 1991). Based on these 
and other data, Fanselow (1991) has recently proposed a model that ascribes differential roles to the dorsal and ventral PAG in the acquisition and expression of aversive Pavlovian conditioning and defensive behavior. In Fanselow's model, the dorsolateral PAG responds directly to noxious stimulation of the body surface and subserves the expression of defensive reactions directed toward these stimuli. Importantly, during aversive Pavlovian conditioning using foot shock as a UCS, the dorsal PAG is necessary for the normal expression of the unconditional reaction (UCR) to shock. Animals with lesions of the dorsolateral PAG show an attenuated UCR to shock while animals with ventral lesions are indistinguishable from intact controls (Fanselow, 1991). The model proposes that the ventral PAG, on the other hand, is directly and selectively responsible for the expression of defensive behavior patterns in response to a signal for shock or to functionally equivalent "innate danger stimuli." This assertion is supported primarily by the observations that lesions of the ventral, but not the dorsal, PAG significantly attenuate freezing behavior in the presence of a CS for shock and that vPAG lesions or local application of opioid antagonists block the expression of antinociceptive CRs in similar situations (Kinscheck et al., 1984; Helmstetter and Landiera-Fernandez, 1990; Fanselow, 1991). Since all lesions in the present study were made after paired exposure to noise and shock, unconditional reactions to shock could not have been affected and thus were not measured. However, the present results do not support a differential role for the dorsal versus the ventral PAG in the expression of hypoalgesia following exposure to the CS since both lesions were equally effective in attenuating CHA.

It is possible that the equivalent degree of disruption observed with dorsal and ventral PAG lesions may have been due to the average relative size of the two lesions. Since the $v P A G$ lesions were normally much smaller than those of the dPAG group, it could be argued that the vPAG plays a more important role in this form of antinociception since a roughly equivalent behavioral effect was seen following different amounts of tissue damage. It is also possible that the dPAG lesions attenuated CHA by interrupting fibers connecting the VPAG with other brain regions (Shipley et al., 1991).

Electrolytic lesions, rather than more selective cytotoxic techniques, were used in the present study as an initial method of assessing the involvement of the PAG and RVM in the expression of these aversive CRs. Because electrolytic lesions destroy local cells as well as axons passing through the lesions site the usc of this technique leaves open the possibility that some or all of the lesion-induced deficits observed in this study could be due to incidental damage rather than destruction of neurons in the target area. By the same reasoning, however, this technique should maximize the chances of seeing any behavioral deficits since both local neurons and critical nonlocal structures could potentially be affected. In the case of the vPAG and medial RVM there is a considerable amount of converging data to suggest that local neurons in these areas participate in various forms of antinociception including CHA (e.g., Watkins et al., 1982, 1983; Helmstetter and Landiera-Fernandez, 1990). Our failure to observe any significant attenuation of ABP CRs following electrolytic lesions of the PAG or RVM is consistent with the results obtained by LeDoux et al. (1988) using both electrolytic and ibotenic acid lesions of the PAG and would suggest that any subsequent studies using excitotoxic lesions in the same regions of the rostral medulla will produce the same outcome. Studies currently underway in our laboratory using ibotenic acid lesions are evaluating the possibility that any of the positive results obtained here are not due to loss of intrinsic neurons.

In conclusion, the results of the present study are consistent with the proposal that the performance of $\mathrm{CHA}$ in the rat relies on a serial neural circuit which includes nociceptive modulatory neurons in the PAG and medial RVM. Furthermore, while significant elevations in ABP may be simultaneously observed with hypoalgesia, lesions that block hypoalgesia do not affect the performance of ABP CRs. The use of this simple preparation in future studies will allow a more detailed analysis of the neural circuits that subserve environmentally mediated forms of hypoalgesia and conditional fear.

\section{References}

Adams DB (1979) Brain mechanisms of offense, defense and submission. Behav Brain Sci 2:201-241.

Bandler R (1988) Brain mechanisms of aggression as revealed by electrical and chemical stimulation: suggestion of a central role for the midbrain periaqueductal grey region. In: Progress in psychobiology and physiological psychology (Epstein AN, Morrison R, eds), pp 67-154. New York: Academic.

Basbaum AI, Fields HL (1984) Endogenous pain control systems: brainstem spinal pathways and endorphin circuitry. Annu Rev Neurosci 7:309-338.

Besson JM, Fardin V, Oliveras JL (1991) Analgesia produced by stimulation of the periaqueductal gray matter: true antinociceptive effects versus stress effects. In: The midbrain periaqueductal gray matter: functional, anatomical and ncurochemical organization (Depaulis A, Bandler R, eds), pp 121-138. New York: Plenum.

Blanchard RJ, Blanchard DC (1988) Ethoexperimental approaches to the biology of emotion. Annu Rev Psychol 39:43-68.

Cannon JT, Prieto GJ, Lee A, Liebeskind JC (1982) Evidence for opioid and non-opioid forms of stimulation-produced analgesia in the rat. Brain Res 243:315-321.

Davis M (1991) The role of the amygdala in conditioned fear. In: The amygdala: neurobiological aspects of emotion, memory and mental dysfunction (Aggleton JP, ed). New York: Wiley-Liss.

Depaulis A, Keay KA, Bandler R (1992) Longitudinal neuronal organization of defensive reactions in the midbrain periaqueductal gray region of the rat. Exp Brain Res 90:307-318.

Fanselow MS (1986) Conditioned fear-induced opiate analgesia: a competing motivational statc theory of stress induced analgesia. In: Stress-induced analgesia (Kelly D, ed). New York: New York Academy of Science.

Fanselow MS (1991) The midbrain periaqueductal gray as a coordinator of action in response to fear and anxiety. In: The midbrain periaqueductal gray matter: functional, anatomical and neurochemical organization (Depaulis A, Bandler R, eds), pp 151-174. New York: Plenum.

Fardin V, Oliveras JL, Besson JM (1984) A reinvestigation of the analgesic effects induced by stimulation of the periaqueductal gray matter in the rat. I. The production of behavioral side effects along with analgesia. Brain Res 306:105-123.

Fields HL, Bry J, Hentall I, Zorman G (1983) The activity of neurons in the rostral medulla of the rat during withdrawal from noxious heat. J Neurosci 3:2345-2552.

Hays WL (1988) Statistics, 4th ed. New York: Holt Rinehart \& Winston.

Helmstetter FJ (1992) The amygdala is essential for the expression of conditional hypoalgesia. Behav Neurosci 106:518-528.

Helmstetter FJ, Bellgowan PS (1993) Lesions of the amygdala block conditional hypoalgesia on the tail flick test. Brain Res 612:253-257.

Helmstetter FJ, Fanselow MS (1987) Effects of naltrexone on learning and performance of conditional fear-induced freezing and opioid analgesia. Physiol Behav 39:501-505.

Helmstetter FJ, Landeira-Fernandez J (1990) Conditional hypoalgesia is attenuated by naltrexone applied to the PAG. Brain Res 537:8892.

Helmstetter FJ, Bellgowan PS, Tershner SA (1993) Inhibition of the tail flick reflex following microinjection of morphinc into the amygdala. Neuroreport 4:471-474. 
Iwata J, LeDoux JE (1988) Dissociation of associative and nonassociative concommitants of classical fear conditioning in the freely behaving rat. Behav Neurosci 102:66-76.

Iwata J, Chida K, LeDoux JE (1987) Cardiovascular responses elicited by stimulation of neurons in the central amygdaloid nucleus in awake but not anesthetized rats resemble conditioned emotional responses. Brain Res 418:183-188.

Kinscheck IB, Watkins LR, Mayer DJ (1984) Fear is not critical to classically conditioned analgesia: the effects of periaqueductal gray lesions and administration of chlordiazepoxide. Brain Res 298:3344.

LeDoux JE, Iwata J, Cicchetti P, Reis DJ (1988) Different projections of the central amygdaloid nucleus mediatc autonomic and behavioral correlates of conditioned fear. J Neurosci 8:2517-2529.

Liebman JM, Mayer DJ, Liebeskind JC (1970) Mesencephalic central gray lesions and fear-motivated behavior in rats. Brain Res 23:353370.

Lovick TA (1985) Ventrolateral medullary lesions block the antinociceptive and cardiovascular responses elicited by stimulating the dorsal periaqueductal gray matter in rats. Pain 21:241-252.

Lovick TA (1991) Interactions between descending pathways from the dorsal and ventrolateral periaqueductal gray matter in the rat. In: The midbrain periaqueductal gray matter: functional, anatomical and neurochemical organization (Depaulis A, Bandler R, eds), pp 101120. New York: Plenum.

Morgan MM, Fields HL (1993) Activity of nociceptive modulatory neurons in the rostral ventromedial medulla associated with volume expansion-induced antinociception. Pain 52:1-9.

Morgan MM, Heinricher MM, Fields HL (1992) Circuitry linking opioid-sensitive noiceptive modulatory systems in periaqueductal gray and spinal cord with rostral ventromedial medulla. Neuroscience 47 : 863-871.
Paxinos G, Watson C (1986) The rat brain in stereotaxic coordinates, 2d ed. New York: Academic.

Randich A, Gebhart GF (1992) Vagal afferent modulation of nociception. Brain Res Rev 17:77-99.

Randich A, Maixner W (1984) Interactions between cardiovascular and pain regulatory systems. Neurosci Biol Behav Rev 8:343-367.

Sandkuhler J, Willman E, Fu QG (1991) Characteristics of midbrain control of spinal nociceptive neurons and nonsomatosensory parameters in the pentobarbital-anethetized rat. J Neurophysiol 65:33-48.

Shipley MT, Ennis M, Rizvi TA, Behbehani M (1991) Topographical specificity of forebrain inputs to the midbrain periaqueductal gray: evidence for discrete longitudinally organized input columns. In: The midbrain periaqueductal gray matter: functional, anatomical and neurochemical organization (Depaulis A, Bandler R, eds), pp 417-448. New York: Plenum.

Thurston CL, Randich A (1992) Effects of vagal afferent stimulation on ON and OFF cells in the rostroventral medulla: relationships to nociception and arterial blood pressure. J Neurophysiol 67:180-196.

Verberne AJM, Guyenet PG (1992) Midbrain central gray: influence on medullary sympathoexcitatory neurons and the baroreflex in rats. Am J Physiol 263:R24-R33.

Watkins LR, Cobelli DA, Mayer DJ (1982) Classical conditioning of front paw and hind paw footshock induced analgesia (FSIA): naloxone reversibility and descending pathways. Brain Res 243:119-132.

Watkins LR, Young EG, Kinscheck IB, Mayer DJ (1983) The neural basis of footshock analgesia: the role of specific ventral medullary nuclei. Brain Res 276:305-315.

Watkins LR, Thurston CL, Fleshner M (1990) Phenylephrine-induced antinociception: investigations of potential neural and endocrine bases. Brain Res 528:273-284. 